\author{
M.A. Ashyralyeva ${ }^{1}$, M. Ashyralyev ${ }^{2}$ \\ ${ }^{1}$ Magtymguly Turkmen State University, Ashgabat, Turkmenistan; \\ ${ }^{2}$ Bahcesehir University, Istanbul, Turkey \\ (E-mail: ashyrmaral2010@mail.ru)
}

\title{
On the numerical solution of identification hyperbolic-parabolic problems with the Neumann boundary condition
}

In the present study, a numerical study for source identification problems with the Neumann boundary condition for a one-dimensional hyperbolic-parabolic equation is presented. A first order of accuracy difference scheme for the numerical solution of the identification problems for hyperbolic-parabolic equations with the Neumann boundary condition is presented. This difference scheme is implemented for a simple test problem and the numerical results are presented.

Keywords: source identification problem, hyperbolic-parabolic differential equations, difference schemes.

\section{Introduction}

Partial differential equations with unknown source terms are widely used in mathematical modeling of reallife systems in many different fields of science and engineering. They have been studied extensively by many researchers (see [1-15] and the references therein).

Various local and nonlocal boundary value problems for hyperbolic-parabolic equations with unknown sources can be reduced to the boundary value problem for the differential equation with parameter $p$

$$
\left\{\begin{array}{l}
u^{\prime \prime}(t)+A u(t)=p+f(t), \quad 0<t<1 \\
u^{\prime}(t)+A u(t)=p+g(t), \quad-1<t<0 \\
u(0+)=u(0-), \quad u^{\prime}(0+)=u^{\prime}(0-) \\
u(-1)=\varphi, \quad u(\lambda)=\psi, \quad-1<\lambda \leq 1
\end{array}\right.
$$

in a Hilbert space $H$ with self-adjoint positive definite operator $A$. The solvability of problem (1) in the space $C(H)$ of continuous $H$-valued functions $u(t)$ defined on $[-1,1]$, equipped with the norm $\|u\|_{C(H)}=\max _{-1 \leq t \leq 1}\|u(t)\|_{H}$, was investigated in [16]. In applications, the stability inequalities for the solution of three source identification problems for hyperbolic-parabolic equations were obtained.

The first and second order of accuracy stable difference scheme for the approximate solution of problem (1) were constructed and investigated in [17] and [18], respectively. The stability estimates for the approximate solutions of two source identification problems for hyperbolic-parabolic equations were obtained.

In this paper we consider the boundary value problem for hyperbolic-parabolic equations

$$
\left\{\begin{array}{l}
u_{t t}-\left(a(x) u_{x}\right)_{x}+\delta u=p(x)+f(t, x), \quad 0<x<1,0<t<1 \\
u_{t}-\left(a(x) u_{x}\right)_{x}+\delta u=p(x)+g(t, x), \quad 0<x<1, \quad-1<t<0 \\
u(0+, x)=u(0-, x), u_{t}(0+, x)=u_{t}(0-, x), \quad 0 \leq x \leq 1 \\
u(-1, x)=\varphi(x), u(1, x)=\psi(x), \quad 0 \leq x \leq 1 \\
u_{x}(t, 0)=u_{x}(t, 1)=0, \quad-1 \leq t \leq 1
\end{array}\right.
$$

where $p(x)$ is an unknown source term. Problem (2) has a unique smooth solution $\{u(t, x), p(x)\}$ for the smooth functions $a(x), \varphi(x), \psi(x), f(t, x), g(t, x)$ and positive constant $\delta$. Note that the boundary value problem (2) can be reduced to the abstract boundary value problem (1) in a Hilbert space $H=L_{2}[0,1]$ with a self-adjoint positive definite operator $A^{x}$ defined by formula $A^{x} u(x)=-\left(a(x) u_{x}\right)_{x}+\delta u$ with domain $D\left(A^{x}\right)=\left\{u(x): u(x), u_{x}(x),\left(a(x) u_{x}\right)_{x} \in L_{2}[0,1], u_{x}(0)=u_{x}(1)=0\right\}$.

We construct the first order of accuracy difference schemes for approximate solutions of boundary value problem (2). We discuss the numerical procedure for implementation of this scheme on the computer. We provide with numerical illustration for simple test problem. 
Numerical procedure for problem (2)

The solution of problem (2) can be written as following:

$$
u(t, x)=v(t, x)+z(x), \quad 0 \leq x \leq 1, \quad-1 \leq t \leq 1,
$$

where $z(x)$ is the solution of problem

$$
\left\{\begin{array}{l}
-\left(a(x) z^{\prime}(x)\right)^{\prime}+\delta z(x)=p(x), \quad 0<x<1 \\
z^{\prime}(0)=z^{\prime}(1)=0
\end{array}\right.
$$

and $v(t, x)$ is the solution of boundary value problem

$$
\left\{\begin{array}{l}
v_{t t}-\left(a(x) v_{x}\right)_{x}+\delta v=f(t, x), \quad 0<x<1,0<t<1 \\
v_{t}-\left(a(x) v_{x}\right)_{x}+\delta v=g(t, x), \quad 0<x<1, \quad-1<t<0 \\
v(0+, x)=v(0-, x), v_{t}(0+, x)=v_{t}(0-, x), \quad 0 \leq x \leq 1 \\
v(1, x)-v(-1, x)=\psi(x)-\varphi(x), \quad 0 \leq x \leq 1 \\
v_{x}(t, 0)=v_{x}(t, 1)=0, \quad-1 \leq t \leq 1 .
\end{array}\right.
$$

Note that from (2)-(4) we get

$$
p(x)=\left(a(x) v_{x}(1, x)\right)_{x}-\delta v(1, x)-\left(a(x) \psi^{\prime}(x)\right)^{\prime}+\delta \psi(x), \quad 0<x<1 .
$$

Taking into account all of the above, the following numerical algorithm can be used for approximate solutions of the boundary value problem (2):

1. Obtain approximate solutions of the boundary value problem (5);

2. Approximate the source $p(x)$ by using (6);

3. Obtain approximate solutions of the boundary value problem (4);

4. Obtain approximate solutions of the boundary value problem (2) by using (3).

The first step of the algorithm

Let $\tau=1 / N$ and $h=1 / M$. We define the grid points $x_{n}=n h, 0 \leq n \leq M$ and $t_{k}=k \tau,-N \leq k \leq N$. For the approximate solutions of the boundary value problem (5) we construct the first order of accuracy difference scheme in $t$

$$
\left\{\begin{array}{c}
\frac{v_{n}^{k-1}-2 v_{n}^{k}+v_{n}^{k+1}}{\tau^{2}}-\frac{1}{h}\left(a\left(x_{n+\frac{1}{2}}\right) \frac{v_{n+1}^{k+1}-v_{n}^{k+1}}{h}-a\left(x_{n-\frac{1}{2}}\right) \frac{v_{n}^{k+1}-v_{n-1}^{k+1}}{h}\right)+\delta v_{n}^{k+1}= \\
=f\left(t_{k+1}, x_{n}\right), \quad 1 \leq k \leq N-1, \quad 1 \leq n \leq M-1 ; \\
\frac{v_{n}^{k}-v_{n}^{k-1}}{\tau}-\frac{1}{h}\left(a\left(x_{n+\frac{1}{2}}\right) \frac{v_{n+1}^{k}-v_{n}^{k}}{h}-a\left(x_{n-\frac{1}{2}}\right) \frac{v_{n}^{k}-v_{n-1}^{k}}{h}\right)+\delta v_{n}^{k}=g\left(t_{k}, x_{n}\right) ; \\
-N+1 \leq k \leq 0, \quad 1 \leq n \leq M-1 ; \\
\frac{v_{n}^{1}-v_{n}^{0}}{\tau}-\frac{1}{h}\left(a\left(x_{n+\frac{1}{2}}\right) \frac{v_{n+1}^{0}-v_{n}^{0}}{h}-a\left(x_{n-\frac{1}{2}}\right) \frac{v_{n}^{0}-v_{n-1}^{0}}{h}\right)+\delta v_{n}^{0}=g\left(t_{0}, x_{n}\right), 1 \leq n \leq M-1 ; \\
v_{n}^{N}-v_{n}^{-N}=\psi\left(x_{n}\right)-\varphi\left(x_{n}\right), \quad 0 \leq n \leq M ; \\
v_{1}^{k}=v_{0}^{k}, \quad v_{M}^{k}=v_{M-1}^{k}, \quad-N \leq k \leq N,
\end{array}\right.
$$

where $v_{n}^{k}$ denotes the numerical approximation of $v(t, x)$ at $\left(t_{k}, x_{n}\right)$. Note that $(7)$ is the second order of accuracy scheme in $x$.

\section{The second step of the algorithm}

Once the numerical solution of the boundary value problem (5) is computed, we use (6) to approximate the source $p(x)$ at grid points as following: 


$$
\begin{gathered}
p_{n}=\frac{1}{h}\left(a\left(x_{n+\frac{1}{2}}\right) \frac{v_{n+1}^{N}-v_{n}^{N}}{h}-a\left(x_{n-\frac{1}{2}}\right) \frac{v_{n}^{N}-v_{n-1}^{N}}{h}\right)-\delta v_{n}^{N}- \\
-\frac{1}{h}\left(a\left(x_{n+\frac{1}{2}}\right) \frac{\psi\left(x_{n+1}\right)-\psi\left(x_{n}\right)}{h}-a\left(x_{n-\frac{1}{2}}\right) \frac{\psi\left(x_{n}\right)-\psi\left(x_{n-1}\right)}{h}\right)+\delta \psi\left(x_{n}\right), \quad n=1,2, \ldots, M-1 .
\end{gathered}
$$

The third step of the algorithm

For the approximate solutions of boundary value problem (4) we have

$$
\left\{\begin{array}{l}
-\frac{1}{h}\left(a\left(x_{n+\frac{1}{2}}\right) \frac{z_{n+1}-z_{n}}{h}-a\left(x_{n-\frac{1}{2}}\right) \frac{z_{n}-z_{n-1}}{h}\right)+\delta z_{n}=p_{n}, \quad n=1,2, \ldots, M-1 ; \\
z_{1}=z_{0}, z_{M}=z_{M-1} .
\end{array}\right.
$$

Solving this system for $z_{0}, z_{1}, z_{2}, \ldots, z_{M}$ and then using (3), we finally obtain the approximate solutions of boundary value problem (2)

$$
u_{n}^{k}=v_{n}^{k}+z_{n}, \quad n=0,1, \ldots, M, \quad k=-N+1, \ldots, N-1 .
$$

\section{Numerical Illustration}

We consider the initial-boundary value problem

$$
\left\{\begin{array}{l}
u_{t t}-u_{x x}+u=p(x)+\left(\left(\pi^{2}+2\right) e^{-t}-1\right) \cos \pi x, \quad 0<x<1,0<t<1 \\
u_{t}-u_{x x}+u=p(x)+\left(\pi^{2} e^{-t}-1\right) \cos \pi x, \quad 0<x<1,-1<t<0 \\
u(0+, x)=u(0-, x), u_{t}(0+, x)=u_{t}(0-, x), \quad 0 \leq x \leq 1 \\
u(-1, x)=e^{1} \cos \pi x, \quad u(1, x)=e^{-1} \cos \pi x, \quad 0 \leq x \leq 1 \\
u_{x}(t, 0)=u_{x}(t, 1)=0, \quad-1 \leq t \leq 1
\end{array}\right.
$$

The exact solution of the problem (9) is

$$
u(t, x)=e^{-t} \cos \pi x, \quad 0 \leq x \leq 1,-1 \leq t \leq 1
$$

with the source term $p(x)=\cos \pi x, 0<x<1$.

The first order of accuracy auxiliary difference scheme (7) for the initial-boundary value problem (9) has the following form

$$
\left\{\begin{array}{l}
\frac{v_{n}^{k-1}-2 v_{n}^{k}+v_{n}^{k+1}}{\tau^{2}}-\frac{v_{n-1}^{k+1}-2 v_{n}^{k+1}+v_{n+1}^{k+1}}{h^{2}}+v_{n}^{k+1}=\left(\left(\pi^{2}+2\right) e^{-t_{k+1}}-1\right) \cos \pi x_{n} \\
1 \leq k \leq N-1, \quad 1 \leq n \leq M-1 ; \\
\frac{v_{n}^{k}-v_{n}^{k-1}}{\tau}-\frac{v_{n-1}^{k}-2 v_{n}^{k}+v_{n+1}^{k}}{h^{2}}+v_{n}^{k}=\left(\pi^{2} e^{-t_{k}}-1\right) \cos \pi x_{n} \\
-N+1 \leq k \leq 0, \quad 1 \leq n \leq M-1 ; \\
\frac{v_{n}^{1}-v_{n}^{0}}{\tau}-\frac{v_{n-1}^{0}-2 v_{n}^{0}+v_{n+1}^{0}}{h^{2}}+v_{n}^{0}=\left(\pi^{2} e^{-t_{0}}-1\right) \cos \pi x_{n}, \quad 1 \leq n \leq M-1 ; \\
v_{n}^{N}-v_{n}^{-N}=\left(e^{-1}-e^{1}\right) \cos \pi x_{n}, \quad 0 \leq n \leq M \\
v_{1}^{k}-v_{0}^{k}=v_{M}^{k}-v_{M-1}^{k}=0, \quad-N \leq k \leq N
\end{array}\right.
$$

which can be written in the matrix form

$$
\left\{\begin{array}{l}
A V_{n+1}+B V_{n}+C V_{n-1}=\phi_{n}, \quad 1 \leq n \leq M-1 \\
V_{1}=V_{0}, \quad V_{M}=V_{M-1}
\end{array}\right.
$$


where

$$
\begin{aligned}
& A=C=\left[\begin{array}{cccccccccc}
0 & 0 & 0 & \cdots & 0 & 0 & 0 & 0 & \cdots & 0 \\
0 & a & 0 & \cdots & 0 & 0 & 0 & 0 & \cdots & 0 \\
0 & 0 & a & \cdots & 0 & 0 & 0 & 0 & \cdots & 0 \\
\vdots & \vdots & \vdots & \ddots & \vdots & \vdots & \vdots & \vdots & & \vdots \\
0 & 0 & 0 & \cdots & a & 0 & 0 & 0 & \cdots & 0 \\
0 & 0 & 0 & \cdots & a & 0 & 0 & 0 & \cdots & 0 \\
0 & 0 & 0 & \cdots & 0 & 0 & b & 0 & \cdots & 0 \\
0 & 0 & 0 & \cdots & 0 & 0 & 0 & b & \cdots & 0 \\
\vdots & \vdots & \vdots & & \vdots & \vdots & \vdots & \vdots & \ddots & \vdots \\
0 & 0 & 0 & \cdots & 0 & 0 & 0 & 0 & \cdots & b
\end{array}\right]_{(2 N+1) \times(2 N+1)} \\
& B=\left[\begin{array}{ccccccccccccc}
-1 & 0 & 0 & \cdots & 0 & 0 & 0 & 0 & 0 & \cdots & 0 & 0 & 1 \\
-1 & c & 0 & \cdots & 0 & 0 & 0 & 0 & 0 & \cdots & 0 & 0 & 0 \\
0 & -1 & c & \cdots & 0 & 0 & 0 & 0 & 0 & \cdots & 0 & 0 & 0 \\
\vdots & \vdots & \vdots & \ddots & \ddots & \vdots & \vdots & \vdots & \vdots & & \vdots & \vdots & \vdots \\
0 & 0 & 0 & \cdots & -1 & c & 0 & 0 & 0 & \cdots & 0 & 0 & 0 \\
0 & 0 & 0 & \cdots & 0 & \sigma & 1 & 0 & 0 & \cdots & 0 & 0 & 0 \\
0 & 0 & 0 & \cdots & 0 & 1 & -2 & d & 0 & \cdots & 0 & 0 & 0 \\
0 & 0 & 0 & \cdots & 0 & 0 & 1 & -2 & d & \cdots & 0 & 0 & 0 \\
\vdots & \vdots & \vdots & & \vdots & \vdots & \vdots & \vdots & & \ddots & \ddots & \ddots & \\
0 & 0 & 0 & \cdots & 0 & 0 & 0 & 0 & 0 & \cdots & 1 & -2 & d
\end{array}\right]_{(2 N+1) \times(2 N+1)} \\
& V_{n}=\left[\begin{array}{c}
v_{n}^{-N} \\
v_{n}^{-N+1} \\
v_{n}^{-N+2} \\
\vdots \\
v_{n}^{0} \\
v_{n}^{1} \\
v_{n}^{2} \\
v_{n}^{3} \\
\vdots \\
v_{n}^{N}
\end{array}\right]_{(2 N+1) \times 1} \\
& \phi_{n}=\left[\begin{array}{c}
\left(e^{-1}-e^{1}\right) \cos \pi x_{n} \\
\tau\left(\pi^{2} e^{-t_{-N+1}}-1\right) \cos \pi x_{n} \\
\tau\left(\pi^{2} e^{-t_{-N+2}}-1\right) \cos \pi x_{n} \\
\vdots \\
\tau\left(\pi^{2} e^{-t_{0}}-1\right) \cos \pi x_{n} \\
\tau\left(\pi^{2} e^{-t_{0}}-1\right) \cos \pi x_{n} \\
\tau^{2}\left(\left(\pi^{2}+2\right) e^{-t_{2}}-1\right) \cos \pi x_{n} \\
\tau^{2}\left(\left(\pi^{2}+2\right) e^{-t_{3}}-1\right) \cos \pi x_{n} \\
\vdots \\
\tau^{2}\left(\left(\pi^{2}+2\right) e^{-t_{N}}-1\right) \cos \pi x_{n}
\end{array}\right]_{(2 N+1) \times 1}
\end{aligned}
$$

with $a=-\frac{\tau}{h^{2}}, b=-\frac{\tau^{2}}{h^{2}}, c=1+\frac{2 \tau}{h^{2}}+\tau, d=1+\frac{2 \tau^{2}}{h^{2}}+\tau^{2}$ and $\sigma=-1+\frac{2 \tau}{h^{2}}+\tau$. To solve the matrix equation (11), we use the modified Gauss elimination method [19]. We seek the solution of the matrix equation (11) by the following form:

$$
\left\{\begin{array}{l}
V_{n}=\alpha_{n+1} V_{n+1}+\beta_{n+1}, \quad n=M-1, \ldots, 2,1 ; \\
V_{M}=\left(I-\alpha_{M}\right)^{-1} \beta_{M},
\end{array}\right.
$$

where $I$ is a $(2 N+1) \times(2 N+1)$ identity matrix, $\alpha_{n}(1 \leq n \leq M)$ are $(2 N+1) \times(2 N+1)$ square matrices and $\beta_{n}(1 \leq n \leq M)$ are $(2 N+1) \times 1$ column vectors, calculated as

$$
\left\{\begin{array}{l}
\alpha_{n+1}=-\left(B+C \alpha_{n}\right)^{-1} A ; \\
\beta_{n+1}=\left(B+C \alpha_{n}\right)^{-1}\left(\phi_{n}-C \beta_{n}\right)
\end{array}\right.
$$

for $n=1,2, \ldots, M-1$. Here $\alpha_{1}$ is an identity matrix and $\beta_{1}$ is a zero vector. 
The numerical solutions are computed using the first order of accuracy scheme (10) for different values of $M$ and $N$. With the obtained numerical solutions we approximate the source $p(x)$ at grid points as following:

$$
p_{n}=\frac{v_{n-1}^{N}-2 v_{n}^{N}+v_{n+1}^{N}}{h^{2}}-v_{n}^{N}-e^{-1} \frac{\cos \pi x_{n-1}-2 \cos \pi x_{n}+\cos \pi x_{n+1}}{h^{2}}+e^{-1} \cos \pi x_{n}, n=1, \ldots, M-1 .
$$

Finally, solving the system

$$
\left\{\begin{array}{l}
-\frac{z_{n-1}-2 z_{n}+z_{n+1}}{h^{2}}+z_{n}=p_{n}, \quad n=1,2, \ldots, M-1, \\
z_{1}=z_{0}, z_{M}=z_{M-1} .
\end{array}\right.
$$

for $z_{0}, z_{1}, z_{2}, \ldots, z_{M}$ and then using (8), we obtain the numerical solutions of problem (9).

We compute the error between the exact solution of problem (9) and corresponding numerical solution by

$$
\left\|E_{u}\right\|_{\infty}=\max _{-N<k<N, 0 \leq n \leq M}\left|u\left(t_{k}, x_{n}\right)-u_{n}^{k}\right|, \quad\left\|E_{p}\right\|_{\infty}=\max _{0<n<M}\left|p\left(x_{n}\right)-p_{n}\right|
$$

where $u\left(t_{k}, x_{n}\right)$ is the exact value of $u(t, x)$ at $\left(t_{k}, x_{n}\right)$ and $p\left(x_{n}\right)$ is the exact value of source $p(x)$ at $x=x_{n} ; u_{n}^{k}$ and $p_{n}$ represent the corresponding numerical solutions. Table shows the errors between the exact solution of the problem (9) and the numerical solutions computed by using the first order of accuracy scheme for different values of $M$ and $N$. We observe that the scheme has the first order convergence as it is expected to be.

Table

The errors between the exact solution of the problem (9) and the numerical solutions computed by using the first order of accuracy difference scheme for different values of $h=1 / M$ and $\tau=1 / N$

\begin{tabular}{|l|c|c|c|c|}
\hline & $\left\|E_{p}\right\|_{\infty}$ & Order & $\left\|E_{u}\right\|_{\infty}$ & Order \\
\hline$N=M=20$ & $1.3246 \times 10^{-1}$ & - & $2.1520 \times 10^{-1}$ & - \\
\hline$N=M=40$ & $7.4275 \times 10^{-2}$ & 0.8346 & $1.1722 \times 10^{-1}$ & 0.8764 \\
\hline$N=M=80$ & $4.0124 \times 10^{-2}$ & 0.8884 & $6.1093 \times 10^{-2}$ & 0.9402 \\
\hline$N=M=160$ & $2.1234 \times 10^{-2}$ & 0.9181 & $3.1190 \times 10^{-2}$ & 0.9699 \\
\hline$N=M=320$ & $1.1072 \times 10^{-2}$ & 0.9394 & $1.5758 \times 10^{-2}$ & 0.9850 \\
\hline
\end{tabular}

\section{Conclusion}

In the present study, the numerical study for source identification problems with the Neumann boundary condition for a one-dimensional hyperbolic-parabolic equation has been conducted. In particular, the first order of accuracy difference schemes for the approximate solutions of the boundary value problem (2) has been constructed and the numerical algorithm for implementation of this scheme has been presented. Numerical example has been provided.

Finally, we note that the second order of accuracy difference schemes for the approximate solutions of boundary value problem (2) can be constructed and implemented in the similar way.

\section{References}

1 Dehghan, M. (2001). Applied Numerical Mathematics, 37, 489-502.

2 Kimura, T., \& Suzuki, T. (1993). SIAM Journal on Applied Mathematics, 53, 1747-1761.

3 Gryazin, Y.A., Klibanov, M.V., \& Lucas, T.R. (1999). Inverse Problems, 25, 373-397.

4 Eidelman, Y.S. (1984). Boundary Value Problems for Differential Equations with Parameters, Voronezh State University, PhD thesis,

5 Ashyralyev, A. (2011). Ukrainian Mathematical Journal, 62, 9, 1397-1408.

6 Orlovskii, D.G., \& Piskarev, S.I. (2013). Differential Equations, 49, 7, 895-907.

7 Ashyralyyev, Ch., \& Akkan, Y. (2015). Electronic Journal of Differential Equations, 2015:188, 1-15.

8 Ashyralyev, A., \& Ashyralyyev, Ch. (2014). Nonlinear Analysis: Modelling and Control, 19, 3, 350-266.

9 Erdogan, A.S., \& Ashyralyev, A. (2014). Applied Mathematics and Computation, 226, 212-228. 
10 Ashyralyev, A., Erdogan, A.S., \& Demirdag, O. (2012). Applied Numerical Mathematics, 62, 1672-1683.

11 Kabanikhin, S.I. (2015). Journal of Inverse and Ill-posed Problems, 12, 5, 519-527.

12 Sazaklioglu, A.U., Ashyralyev, A., \& Erdogan, A.S. (2017). Filomat, 31, 4, 1057-1064.

13 Ashyralyev, A., \& Sazaklioglu, A.U. (2017). Numerical Functional Analysis and Optimization, 38, 10, 1276-1294.

14 Orazov, I., \& Sadybekov, M.A. (2012). Siberian Mathematical Journal, 53, 146-151.

15 Aliev, F., Ismailov, N., \& Namazov, A.A. (2016). Applied and Computational Mathematics, 15, 3, 370-376.

16 Ashyralyev, A., \& Ashyralyyeva, M.A. (2015). Contemporary Analysis and Applied Mathematics, 3, 1, $88-103$.

17 Ashyralyyeva, M.A., \& Ashyralyyev, A. (2015). AIP Conference Proceedings, 1676, 0200241-0200246.

18 Ashyralyyeva, M.A., \& Ashyraliyev, M. (2016). AIP Conference Proceedings, 1759, 0200231-0200236.

19 Samarskii, A.A., \& Nikolaev, E.S. (1989). Numerical Methods for Grid Equations: Iterative Methods. Basel: Birkhauser.

\author{
М.А. Ашыралыева, М. Ашыралыев
}

\title{
Шеттік шарты Нейман түрінде болатын идентификациялық гипербола-параболалық есептерді сандық шешу туралы
}

\begin{abstract}
Шеттік шарты Нейман түріндегі бір өлшемді гипербола-параболалық түрдегі теңдеу үшін көздерді идентификациялау есебін сандық зерттеу нәтижесі ұсынылған. Шеттік шарты Нейман түріндегі гипербола-параболалық түрдегі теңдеулер үшін көздерді идентификациялау есебін сандық шешу үшін дәлдігі бірінші ретті айырымдық формуласы келтірілген. Бұл формула қарапайым есеп үшін пайдаланылған, сонымен қатар сандық есептеулер нәтижесі берілген.

Kiлm сөздер: көздерді идентификациялау есебі, гипербола-параболалық дифференциалды теңдеу, айырымдық схема.
\end{abstract}

\author{
М.А. Ашыралыева, М. Ашыралыев
}

\section{О численном решении идентификационных гиперболо-параболических задач с граничным условием Неймана}

\begin{abstract}
В статье представлено численное исследование задачи идентификации источников с граничным условием Неймана для одномерного гиперболо-параболического уравнения. Представлена разностная схема первого порядка точности для численного решения задач идентификации для гиперболо-параболических уравнений с граничным условием Неймана. Эта разностная схема реализована для простой тестовой задачи.
\end{abstract}

Ключевые слова: задача идентификации источника, гиперболо-параболические дифференциальные уравнения, разностные схемы. 